\title{
Swarming bottom feeders: Flocking at solid-liquid interfaces
}

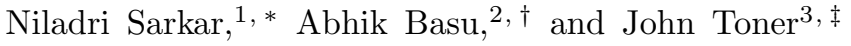 \\ ${ }^{1}$ Instituut-Lorentz, Leiden University, P.O. Box 9506, 2300 RA Leiden, The Netherlands \\ ${ }^{2}$ Condensed Matter Physics Division, Saha Institute of Nuclear Physics, Calcutta 700064, West Bengal, India \\ ${ }^{3}$ Department of Physics and Institute of Theoretical Science, \\ University of Oregon, Eugene, Oregon 97403, USA
}

(Dated: June 28, 2021)

\begin{abstract}
We present the hydrodynamic theory of coherent collective motion ("flocking") at a solid-liquid interface, and many of its predictions for experiment. We find that such systems are stable, and have long-range orientational order, over a wide range of parameters. When stable, these systems exhibit "giant number fluctuations", which grow as the $3 / 4$ th power of the mean number. Stable systems also exhibit anomalous rapid diffusion of tagged particles suspended in the passive fluid along any directions in a plane parallel to the solid-liquid interface, whereas the diffusivity along the direction perpendicular to the plane is not anomalous. In the remaining parameter space, the system becomes unstable.
\end{abstract}

Many "active" systems consist of macroscopically large numbers of self-propelled particles that align their directions of motion. This occurs both in living [1 [5] and synthetic 6-10 systems. Such "active orientationally ordered phases" exhibit many phenomena impossible in their equilibrium analogs (e.g., nematics [1]), including spontaneous breaking of continuous symmetries in two dimensions 12 15, instability in the extreme Stokesian limit 16, and giant number fluctuations [17 19].

"Dry" active systems - i.e., those lacking momentum conservation due to, e.g., friction with a substrate [14, 19, 20- behave quite differently from "wet" active fluids (i.e., those with momentum conservation) [21.

In this paper, we present the first theory of a natural hybrid of these two cases: polar active particles at a solid-liquid interface (see figure (1)). We are motivated by experiments 22] in which highly concentrated actin filaments on a solid-fluid interface are propelled by motor proteins, and those of Bricard et al [23, 24, who studied the emergence of macroscopically directed motion in "Quincke rollers". The latter are motile colloids, spontaneously rolling on a solid substrate when a sufficiently strong electric field is applied.

These systems differ from both dry and wet active matter, as defined above, by having both friction from the underlying solid substrate and the long range hydrodynamic interactions due to the overlying bulk passive fluid.

The geometry we consider here, as in Ref. 22, 23, places a collection of polar, self-propelled particles at the flat interface (the $x-y$ plane of our coordinate system) between a solid substrate and a semi-infinite bulk isotropic and incompressible passive liquid. as illustrated in Fig. 1. We consider the extreme Stokesian limit, in which inertial forces are completely negligible compared to viscous forces.

\footnotetext{
* niladri2002in@gmail.com

$\dagger$ abhik.123@gmail.com, abhik.basu@saha.ac.in

$\ddagger$ jjt@uoregon.edu
}

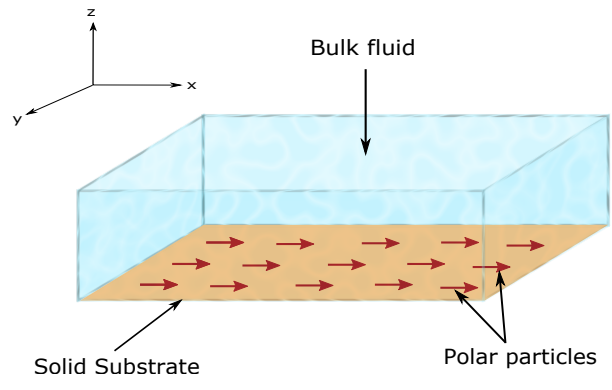

FIG. 1. (Color online) Schematic diagram of our system: a layer of active polar particles moving on a solid substrate with a passive ambient ("bulk") fluid above.

The most surprising result of our work is that, even in the presence of noise, this system can be in a stable, long-range ordered polar state, in sharp contrast to "wet" active systems, which are generically unstable [16 at low Reynolds number, and equilibrium systems, which cannot display long range orientational order in two dimensions at finite temperature 25] 28.

Remarkably, this ordered state is predicted even by a linear theory. Furthermore, this linear theory provides an asymptotically exact long wavelength description, in contrast to dry polar active systems, which can only be correctly described by a non-linear theory. Indeed, dry polar active systems can only exhibit long range order due to non-linear effects [12 15.

Concomitant with the long-range polar order, the density fluctuations are giant: the standard deviation $\sqrt{\left\langle(N-\langle N\rangle)^{2}\right\rangle}$ of the number $N$ of the active particles contained in a fixed open area scales with its average $\langle N\rangle$ according to

$$
\sqrt{\left\langle(N-\langle N\rangle)^{2}\right\rangle} \propto\langle N\rangle^{3 / 4}
$$

This agrees very well with the experiments of [22], which found $\sqrt{\left\langle(N-\langle N\rangle)^{2}\right\rangle} \propto\langle N\rangle^{0.8}$. Note that our prediction should not be confused with qualitatively similar 
predictions for dry active matter [17, 29] and active nematics [30, for which the exponent is different, because they belong to different universality classes.

We also find that the fluctuations in the active fluid layer stir the bulk fluid above it, making the diffusion of a passive tagged particle parallel to the active fluid layer anomalous: specifically, the mean squared displacement grows with time $t$ as $t \ln t$, whereas the diffusive motion perpendicular to the active fluid layer remains conventional, i.e., the mean squared displacement scales like $t$.

To understand the physics of this system, we have constructed a theory which, when linearized for small fluctuations about a uniform reference state, is asymptotically exact in the long wavelength limit, and gives the above results. We define $\hat{\mathbf{p}}\left(\mathbf{r}_{\|}, t\right)$ as the coarse grained polarization of the active particles, and $\rho\left(\mathbf{r}_{\|}, t\right)$ as the conserved areal density of the active polar particles on

the surface. Taking our uniform reference state to be $\hat{\mathbf{p}}\left(\mathbf{r}_{\|}, t\right)=\hat{\mathbf{x}}$ (see Fig. 1), and $\rho=\rho_{0}$, one hydrodynamic variable is the transverse fluctuations $p_{y}$ of $\hat{\mathbf{p}}\left(\mathbf{r}_{\|}, t\right)$, which we take to have unit magnitude, i.e., $|\hat{\mathbf{p}}|^{2}=1$. This is a non-conserved broken symmetry - i.e., "Goldstone" mode. Our second hydrodynamic variable is the fluctuations $\delta \rho\left(\mathbf{r}_{\|}, t\right) \equiv \rho\left(\mathbf{r}_{\|}, t\right)-\rho_{0}$ of the density from its mean value.

These variables couple to the bulk passive fluid velocity $\mathbf{v}\left(\mathbf{r}_{\|}, z, t\right)$ via an active boundary condition given below in (12). Eliminating $\mathbf{v}\left(\mathbf{r}_{\|}, z, t\right)$ by solving the Stokes equation for the bulk fluid subject to this active boundary condition gives the equations of motion for the spatially Fourier transformed fields $p_{y}(\mathbf{q}, t)$ and $\delta \rho(\mathbf{q}, t)$ :

$$
\partial_{t} \delta \rho(\mathbf{q}, t)=-i v_{\rho}\left[q_{x} \delta \rho(\mathbf{q}, t)+\rho_{c} q_{y} p_{y}\right]+i \mathbf{q} \cdot \mathbf{f}_{\rho}(\mathbf{q}, t),
$$

$$
\partial_{t} p_{y}(\mathbf{q}, t)=-i v_{p} q_{x} p_{y}(\mathbf{q}, t)-\gamma\left(\frac{q^{2}+q_{y}^{2}}{q}\right) p_{y}(\mathbf{q}, t)-\left(\frac{\gamma_{\rho}}{\rho_{c}}\right)\left(\frac{q_{x} q_{y}}{q}\right) \delta \rho(\mathbf{q}, t)-i \sigma_{t} q_{y} \delta \rho(\mathbf{q}, t)+f_{y}(\mathbf{q}, t)
$$

where $v_{\rho}, v_{p}, \gamma, \gamma_{\rho}, \rho_{c}$, and $\sigma_{t}$ are parameters of our model. Note the non-analytic character of the damping $\gamma$ and $\gamma_{\rho}$ terms in (3); due to long-ranged hydrodynamic interactions mediated by the bulk passive fluid.

In (2) and (3), $\mathbf{f}_{\rho}$ and $f_{y}$ are zero-mean Gaussian white noises whose variances are parameters of our model.

For stability, fluctuations must decay for all directions of q. We show in the associated long paper (ALP) 31] that this condition is satisfied provided that the analogs of the bulk compressibility and the shear and bulk viscosities in our system are all positive, and that the coupling of the density of the active particles to their self-propelled speeds is not too strong.

Thus, in contrast to "wet" active matter in the "Stokesian" limit [15, 16], our "mixed" system can be generically stable. Indeed, the requirements for stability are almost as easily met for these systems as for an equilibrium fluid. Furthermore, when the stability conditions are met, fluctuations about the uniform ordered state in this model decay with a rate that scales linearly with $q$, quite different from the linear theory of dry active matter. The also propagate nondispersively with a wavespeed independent of $q$.

This unusual damping in this linear theory is responsible for many novel phenomena: most strikingly, it makes $\left\langle p_{y}^{2}\left(\mathbf{r}_{\perp}, t\right)\right\rangle$ asymptotically independent of the lateral size of the system, a tell-tale signature of orientational longrange order. It also leads to giant number fluctuations of the active particles given by (1), as mentioned earlier.

In the ordered state, the active particles "stir" the passive fluid above them. The mean squared components $\left\langle v_{x}^{2}\left(\mathbf{r}_{\|}, z, t\right)\right\rangle$, and $\left\langle v_{y}^{2}\left(\mathbf{r}_{\|}, z, t\right)\right\rangle$ of the passive fluid velocity field $\mathbf{v}\left(\mathbf{r}_{\|}, z, t\right)$ thereby induced are inversely proportional to the distance $z$ from the solid-fluid interface.

The unequal-time correlations $\left\langle v_{x, y}\left(\mathbf{r}_{\|}, z, t\right) v_{x, y}\left(\mathbf{r}_{\|}, z, 0\right)\right\rangle$ of the in-plane velocity fluctuations of the passive fluid also exhibit long temporal correlations, which decay as $1 / t$, whereas the correlation $\left\langle v_{z}\left(\mathbf{r}_{\|}, z, t\right) v_{z}\left(\mathbf{r}_{\|}, z, 0\right)\right\rangle$ of the the bulk fluid velocity perpendicular to the surface decays as $1 / t^{3}$.

The correlations of the in-plane velocity in turn lead to anomalous diffusion of neutrally buoyant passive particles in the $x$ - and $y$-direction, with variances of the displacements growing faster with time than the linear dependence found for simple brownian particles. Specifically, we find, for a particle that is initially a height $z_{0}$ above the solid-liquid interface,:

$$
\left\langle\left(r_{i}(t)-r_{i}(0)\right)^{2}\right\rangle= \begin{cases}2 D_{i} t\left[\ln \left(\frac{v_{0} t}{z_{0}}\right)+O(1)\right], & t \ll \frac{z_{0}^{2}}{D_{z}}, \\ D_{i} t\left[\ln \left(\frac{v_{0}^{2} t}{D_{z}}\right)+O(1)\right], & t \gg \frac{z_{0}^{2}}{D_{z}},\end{cases}
$$

where $i=x, y, v_{0}$ is a system-dependent characteristic speed (roughly speaking, the self-propulsion speed of the active particles), $z_{0}$ is the initial distance from the surface, and $D_{x, y, z}$ are diffusion constants which are independent of $z_{0}$. Note that the mean square displacements depend on the initial height $z_{0}$ for short times $t \ll z_{0}^{2} / D_{z}$, but not for long times $t \gg z_{0}^{2} / D_{z}$. The crossover between these limits is the time $t=z_{0}^{2} / D_{z}$ it takes for a neutrally buyoant particle to diffuse a distance $z_{0}$ in the $z$-direction. 
Diffusion in the $z$-direction remains conventional, controlled by a $z$-independent diffusivity.

This set of predictions could also be tested experimentally by particle tracking of neutrally buoyant tracer particles in the passive fluid.

Particles denser than the passive fluid, which therefore sediment, will also be affected by this activity induced flow. We find that particles sedimenting at a speed $v_{\text {sed }} \ll v_{0}$ from an initial height $z_{0}$ will, when they reach the surface, be spread out over a region of RMS dimensions $\sqrt{\left\langle\left(x(z=0)-x\left(z=z_{0}\right)\right)^{2}\right\rangle}$ and $\sqrt{\left\langle\left(y(z=0)-y\left(z=z_{0}\right)\right)^{2}\right\rangle}$ in the $x$ and $y$ directions, respectively, with

$$
\left\langle\left(r_{i}(t)-r_{i}(0)\right)^{2}\right\rangle=2 D_{i}\left(\frac{z_{0}}{v_{\text {sed }}}\right) \ln \left(\frac{v_{0}}{v_{\text {sed }}}\right), \quad v_{\text {sed }} \ll v_{0},
$$

where $v_{0}$ is roughly the mean speed of the active particles, and $v_{\text {sed }}$ is the speed at which the sedimenting particles sink.

Once again, these predictions should be readily testable in particle tracking experiments.

We find that the polarization $\hat{\mathbf{p}}$, has a simple scaling form for its spatio-temporally Fourier transformed correlation function:

$$
C_{p p}(\mathbf{q}, \omega) \equiv\left\langle\left|p_{y}(\mathbf{q}, \omega)\right|^{2}\right\rangle=\left(\frac{1}{q^{2}}\right) F_{p p}\left(\left(\frac{\omega}{q}\right), \theta_{\mathbf{q}}\right),
$$

where the scaling function $F_{p p}\left(u, \theta_{\mathbf{q}}\right)$ is given in the ALP; and $\theta_{\mathbf{q}} \equiv \tan \left(q_{y} / q_{x}\right)$ is the angle between $\mathbf{q}$ and the direction $\hat{\mathbf{x}}$ of the mean polarization. The positions of the peaks in $C_{p p}(\mathbf{q}, \omega)$ versus $\omega$ [32] (but most definitely not their widths), are precisely those found for dry active matter in 13-15]; i.e., $\omega_{\text {peak }}=c_{ \pm}\left(\theta_{\mathbf{q}}\right) q$, where $c_{ \pm}\left(\theta_{\mathbf{q}}\right)$ is given by (15) and plotted in Figure (2).

These peak positions agree with those found in the experiments of 24] on Quinke rollers.

The density-density correlation function $C_{\rho \rho}(\mathbf{q}, \omega) \equiv$ $\left\langle|\delta \rho(\mathbf{q}, \omega)|^{2}\right\rangle$, and the density-polarization crosscorrelation $C_{p \rho}(\mathbf{q}, \omega) \equiv\left\langle p_{y}(\mathbf{q}, \omega) \delta \rho(-\mathbf{q},-\omega)\right\rangle$, both obey similar scaling laws, which are given in detail in the ALP.

Integrating these spatio-temporally Fouriertransformed correlation functions over all frequencies $\omega$ shows that the equal time correlation functions $C_{p p}(\mathbf{q}) \equiv\left\langle\left|p_{y}(\mathbf{q}, t)\right|^{2}\right\rangle, C_{\rho \rho}(\mathbf{q}) \equiv\left\langle|\delta \rho(\mathbf{q}, t)|^{2}\right\rangle$, and $C_{p \rho}(\mathbf{q}) \equiv\left\langle p_{y}(\mathbf{q}, t) \delta \rho(-\mathbf{q}, t)\right\rangle$ all scale like $1 / q$. Their dependence on the direction of $\mathbf{q}$ is given explicitly in the ALP.

Fourier transforming these in space shows that the real space, equal-time correlation functions $C_{p p}(\mathbf{r})=$ $\left\langle p_{y}(\mathbf{r}+\mathbf{R}, t) p_{y}(\mathbf{R}, t)\right\rangle, C_{\rho \rho}(\mathbf{r}) \equiv\langle\delta \rho(\mathbf{r}+\mathbf{R}, t) \delta \rho(\mathbf{R}, t)\rangle$, and $C_{p \rho}(\mathbf{r}) \equiv\left\langle p_{y}(\mathbf{r}+\mathbf{R}, t) \delta \rho(\mathbf{R}, t)\right\rangle$ all scale like $1 / r$, and depend on the direction of $\mathbf{r}$. Explicit expressions for this direction-dependence are given in the ALP.

These predictions could also be tested experimentally in systems in which the active particles can be imaged, like those of [22, 23]. Although the anisotropy of the

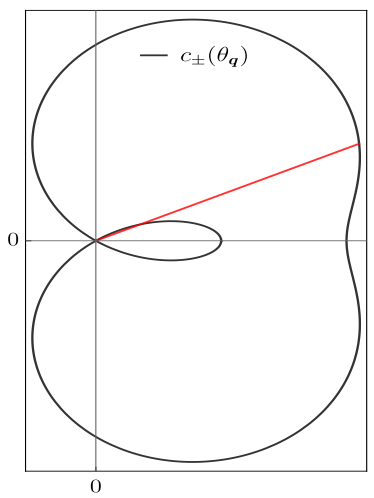

FIG. 2. (Color online) Polar plot of the sound speeds; the polarization points directly to the right. That is, the distance along a straight line line drawn from the origin and making an angle $\theta$ with the $x$-axis to its intersection with the curve is proportional to the sound speed of a mode propagating at the same angle $\theta$ to the mean polarization direction $\hat{\mathbf{x}}$. There are two intersections for each such line, corresponding to the two roots given in equation 15 for the sound speeds. Here we have taken $v_{\rho}=1, v_{p}=c_{0}=2$, and $\gamma=.3$ (all in arbitrary units).

system ensures that all the correlators are anisotropic functions of distance $\mathbf{r}$, nonetheless, their spatial scaling remains isotropic. That is, the anisotropy exponent $\zeta$ that determines the relative scaling between $x$ and $y$ is $\zeta=1$, in contrast to the Toner-Tu model [14].

The correlator $C_{\rho \rho}\left(\mathbf{r}-\mathbf{r}^{\prime}\right)$ can be used to obtain the result (1) for the giant number fluctuations. The bulk velocity can be obtained from $p_{y}(\mathbf{r}, t)$ and $\delta \rho(\mathbf{r}, t)$ through the aforementioned solution of the Stokes equation subject to the active boundary condition. This in turn allows us to derive the anomalous diffusion (4); see the ALP 31] for detailed derivations.

We will now provide an outline of how we obtained these results. Details can be found in the ALP.

In the presence of friction from the substrate, there is no momentum conservation on the surface, so the only conserved variable on the surface is the active particle number. We also include the bulk fluid velocity $\mathbf{v}(\mathbf{r}, t)$, which is defined throughout the semi-infinite three dimensional (3D) space above the surface, since in that space momentum (which is equivalent to velocity in the limit of an incompressible bulk fluid) is conserved. However, we work in the Stokesian limit, in which viscous forces dominate inertial ones.

We formulate the hydrodynamic equations for these variables by expanding their equations of motion phenomenologically in powers of fluctuations of both fields $\hat{\mathbf{p}}$ and $\rho$ from their mean values, and in spatio-temporal gradients. In doing so, we respect all symmetries and conservation laws of the underlying dynamics. In our nonequilibrium system, additional equilibrium constraints like detailed balance do not apply. Our system has underlying rotational invariance in the plane of the surface, which is spontaneously broken by the active parti- 
cles when they align their polarizations.

Conservation of the active particles implies that $\rho\left(\mathbf{r}_{\|}, t\right)$ obeys a continuity equation:

$$
\partial_{t} \rho+\nabla_{s} \cdot \mathbf{J}_{\rho}=0
$$

where $\boldsymbol{\nabla}_{s} \equiv \hat{\mathbf{x}} \partial / \partial x+\hat{\mathbf{y}} \partial / \partial y$ is the $2 \mathrm{D}$ gradient operator, with $\hat{\mathbf{x}}$ and $\hat{\mathbf{y}}$ the unit vectors along the $x$ and $y$ axis respectively. We phenomenologically expand the active particle current $\mathbf{J}_{\rho}$ to leading order in powers of the bulk velocity evaluated at the surface $\mathbf{v}\left(\mathbf{r}_{\|}, z=0\right)$, and gradients, while respecting rotation invariance. In practice, this means we can make the vector $\mathbf{J}_{\rho}$ only out of vectors the system itself chooses, i.e., out of gradients, the surface velocity $\mathbf{v}_{s}\left(\mathbf{r}_{\|}, t\right) \equiv \mathbf{v}\left(\mathbf{r}_{\|}, z=0, t\right)$, and the polarization $\hat{\mathbf{p}}\left(\mathbf{r}_{\|}, t\right)$. These constraints force $\mathbf{J}_{\rho}$ to take the form:

$$
\mathbf{J}_{\rho}\left(\mathbf{r}_{\|}\right)=\rho_{e}\left(\rho,\left|\mathbf{v}_{s}\right|\right) \mathbf{v}_{s}(x, y)+\kappa\left(\rho,\left|\mathbf{v}_{s}\right|\right) \hat{\mathbf{p}}
$$

to leading order in gradients. The factor $\kappa\left(\rho,\left|\mathbf{v}_{s}\right|\right)$ is an active parameter reflecting the self-propulsion of the particles through interaction with the solid substrate, while the $\rho_{e}$ term reflects convection of the active particles by the passive fluid above them. The parameter $\rho_{e} \neq \rho$ in general due to drag between the active particles and the substrate.

In calculating the bulk velocity $\mathbf{v}\left(\mathbf{r}_{\|}, z, t\right)$, we assume the bulk fluid is in the extreme "Stokesian" limit, in which inertia is negligible relative to viscous drag. This should be appropriate for most systems in which the active particles are microscopic, since the Reynolds' number will be extremely low for such particles. It is, how- ever, certainly not valid for bottom-feeding fish, so the title of this paper takes some poetic license!

In this limit, the three-dimensional (3D) incompressible bulk velocity field $\mathbf{v}=\left(v_{i}, v_{z}\right), i=x, y$ satisfies the 3D Stokes' equation

$$
\eta \nabla_{3}^{2} v_{\alpha}\left(\mathbf{r}_{\|}, z\right)=\partial_{\alpha} \Pi\left(\mathbf{r}_{\|}, z\right)
$$

where $\eta$ is the bulk viscosity of the fluid, together with the incompressibility constraint $\boldsymbol{\nabla}_{3} \cdot \mathbf{v}=0$. Here $\boldsymbol{\nabla}_{3} \equiv$ $\hat{\mathbf{x}} \partial / \partial x+\hat{\mathbf{y}} \partial / \partial y+\hat{\mathbf{z}} \partial / \partial z$ is the full three-dimensional gradient operator, with $\hat{\mathbf{x}}, \hat{\mathbf{y}}$, and $\hat{\mathbf{z}}$ as the unit vectors along the $x, y$, and $z$ axes respectively, and $\Pi$ is the bulk pressure which enforces the incompressibility constraint.

This equation (9) can be solved exactly for the bulk velocity $\mathbf{v}\left(\mathbf{r}_{\|}, z, t\right)$ in terms of the surface velocity $\mathbf{v}_{s}\left(\mathbf{r}_{\|}, t\right)$. If we Fourier expand the surface velocity:

$$
\mathbf{v}_{s}\left(\mathbf{r}_{\|}, t\right)=\frac{1}{\sqrt{L_{x} L_{y}}} \sum_{\mathbf{q}} \mathbf{v}_{s}(\mathbf{q}, t) e^{i \mathbf{q} \cdot \mathbf{r}_{\|}}
$$

where $\left(L_{x}, L_{y}\right)$ are the linear dimensions of our (presumed rectangular) surface, then, as we show in the ALP, the bulk velocity $\mathbf{v}\left(\mathbf{r}_{\|}, z, t\right)$ is given by

$$
\mathbf{v}\left(\mathbf{r}_{\|}, z, t\right)=\frac{1}{\sqrt{L_{x} L_{y}}} \sum_{\mathbf{q}}\left[\mathbf{v}_{s}(\mathbf{q}, t)-z\left(\mathbf{q} \cdot \mathbf{v}_{s}\right)(\hat{\mathbf{q}}+i \hat{\mathbf{z}})\right] e^{-q z+i \mathbf{q} \cdot \mathbf{r}_{\perp}}
$$

The last ingredient in our theory is the boundary condition on the bulk fluid velocity at the interface. The active particles at the solid-liquid interface generate active forces, which change the boundary condition from the familiar partial-slip boundary condition to:

$$
v_{s i}\left(\mathbf{r}_{\|}, t\right)=v_{a}(\rho) p_{i}\left(\mathbf{r}_{\|}, t\right)+\zeta_{1}(\rho) \hat{\mathbf{p}} \cdot \nabla_{S} p_{i}+\zeta_{2}(\rho) p_{i} \nabla_{S} \cdot \hat{\mathbf{p}}+p_{i} \hat{\mathbf{p}} \cdot \nabla_{S} \zeta(\rho)+\mu \eta\left(\frac{\partial v_{i}\left(\mathbf{r}_{\|}, z, t\right)}{\partial z}\right)_{z=0}-\partial_{i} P_{s}(\rho)
$$

where $v_{a}(\rho)$ is the spontaneous self-propulsion speed of the active particles relative to the solid substrate, $\zeta_{1,2}$ and $\zeta$ are coefficients of the active stresses permitted by symmetry, and $P_{s}(\rho)$ is a surface osmotic pressure. As before $i=(x, y)$. For a system in thermal equilibrium, $v_{a}=0=\zeta_{1,2}(\rho)=\zeta(\rho)$, and 12 reduces to the well- known equilibrium partial slip boundary condition 33 .

We now turn to the equation of motion for $\hat{\mathbf{p}}$. As the active particles are polar, the system lacks $\hat{\mathbf{p}} \rightarrow-\hat{\mathbf{p}}$ symmetry. This allows $\partial_{t} \hat{\mathbf{p}}$ to contain terms even in $\hat{\mathbf{p}}$. The most general equation of motion for $p_{k}$ allowed by symmetry, neglecting "irrelevant" terms,

$$
\partial_{t} p_{k}=T_{k i}\left(\alpha v_{s i}-\lambda_{p v}\left(\mathbf{v}_{s} \cdot \nabla_{s}\right) p_{i}+\left(\frac{\nu_{1}-1}{2}\right) p_{j} \partial_{i} v_{s j}+\left(\frac{\nu_{1}+1}{2}\right)\left(\hat{\mathbf{p}} \cdot \nabla_{s}\right) v_{s i}-\lambda\left(\hat{\mathbf{p}} \cdot \nabla_{s}\right) p_{i}-\partial_{i} P_{p}(\rho)+f_{i}\right),
$$

where the projection operator $T_{k i} \equiv \delta_{k i}^{s}-p_{k} p_{i}$ insures that the fixed length condition $|\hat{\mathbf{p}}|=1$ on $\hat{\mathbf{p}}$ is preserved.
It is the breaking of Galilean invariance by the solid substrate that allows $\lambda_{p v}$ to differ from 1, and the presence 
of the "self-advection" term $\alpha$ in 13 . The terms proportional to $\nu_{1}$ are "flow alignment terms", identical in form to those found in nematic liquid crystals [34. The term with coefficient $\lambda$ is allowed by the polar symmetry of the particles, and can be interpreted as self advection of the particle polarity in its own direction. The function $P_{p}(\rho)$ is a density dependent "surface polarization pressure" independent of the "osmotic pressure" $P_{s}(\rho)$ introduced earlier. We have also added to the equation of motion (13) a white noise $\mathbf{f}$ with statistics

$$
\left\langle f_{i}\left(\mathbf{r}_{\perp}, t\right) f_{j}\left(\mathbf{r}_{\perp}^{\prime}, t^{\prime}\right)\right\rangle=2 D_{p} \delta_{i j} \delta\left(\mathbf{r}_{\perp}-\mathbf{r}_{\perp}^{\prime}\right) \delta\left(t-t^{\prime}\right) .
$$

Our hydrodynamic model, then, is summarized by the equations of motion (7), (8), and (13) for $\rho$ and $\hat{\mathbf{p}}$, respectively, and the solution (11) of the Stokes equation (9) for the bulk velocity field $\mathbf{v}(x, y, z, t)$ obtained with the boundary condition (12). Fluctuations also involve the noise correlations 14.

These equations of motion and boundary conditions have an obvious spatially uniform, steady state solution: $\rho\left(\mathbf{r}_{\|}, t\right)=\rho_{0}, \hat{\mathbf{p}}\left(\mathbf{r}_{\|}, t\right)=\hat{\mathbf{x}}$, where we have defined $v_{0} \equiv v_{a}\left(\rho_{0}\right)$ and have chosen the $\hat{\mathbf{x}}$ axis of our coordinate system to be along the (spontaneously chosen) direction of polarization, as illustrated in figure (1).

To study fluctuations about this steady state, we expand the equations of motion (7), (8), and (13) for $\rho$ and $\hat{\mathbf{p}}$, and the boundary condition (12), to linear order in $\delta \rho$ and $p_{y}$. We obtain the bulk velocity $\mathbf{v}\left(\mathbf{r}_{\|}, z, t\right)$ from the surface velocity $\mathbf{v}_{s}\left(\mathbf{r}_{\|}, t\right)$ using our solution (11) of the Stokes equation. This ultimately produces Eqs. (2) and (3) , where the phenomenological hydrodynamic parameters $v_{\rho}, v_{p}, \gamma, \gamma, \rho_{c}$, and $\sigma_{t}$ are all related to the expansion coefficients of the various parameters introduced above when expanded in powers of the small fluctuations $\delta \rho$ and $p_{y}$. The rather involved details of this calculation are given in the ALP.
The correlation functions can be straightforwardly determined from these equations of motion, and shown to have peaks at $\omega_{\text {peak }}=c_{ \pm}\left(\theta_{\mathbf{q}}\right) q$, where $c_{ \pm}\left(\theta_{\mathbf{q}}\right)$ is given by

$$
\begin{aligned}
c_{ \pm}\left(\theta_{\mathbf{q}}\right)= & \pm \sqrt{\frac{1}{4}\left(v_{\rho}-v_{p}\right)^{2} \cos ^{2} \theta_{\mathbf{q}}+c_{0}^{2} \sin ^{2} \theta_{\mathbf{q}}} \\
& +\left(\frac{v_{\rho}+v_{p}}{2}\right) \cos \theta_{\mathbf{q}}
\end{aligned}
$$

We have presented a comprehensive hydrodynamic theory of flocking at a solid-liquid interface. This theory makes quantitative, experimentally testable predictions about orientational long range order, spatiotemporal scaling of fluctuations, giant number fluctuations and anomalous diffusion along directions parallel to the solid-liquid interface. These predictions are exact in the asymptotic long wavelength limit, as will be shown in the ALP using renormalization group arguments. One simple variant on our system would be to replace the bulk isotropic fluid of our system with a nematic.

Acknowledgements: One of us (AB) thanks the SERB, DST (India) for partial financial support through the MATRICS scheme [file no.: MTR/2020/000406]. NS is partially supported by Netherlands Organization for Scientific Research (NWO), through the Vidi grant No. 2016/N/00075794. We thank S. Ramaswamy for sharing reference [35] with us. NS thanks Institut Curie and MPIPKS for their support through postdoctoral fellowships while some of this work was being done. AB thanks the MPIPKS, Dresden for their hospitality, and their support through their Visitors' Program, while a portion of this work was underway. JT likewise thanks the MPIPKS for their hospitality, and their support through the Martin Gutzwiller Fellowship, and the Higgs Center of the University of Edinburgh for their support with a Higgs Fellowship.
[1] K. Kruse, J.F. Joanny, F. Jülicher, J. Prost, and K. Sekimoto, Asters, vortices, and rotating spirals in active gels of polar filaments, Phys. Rev. Lett. 92, 078101(2004).

[2] K. Kruse, J.F. Joanny, F. Jülicher, J. Prost, and K. Sekimoto, Generic theory of active polar gels: a paradigm for cytoskeletal dynamics, Eur. Phys. J. E, 16, 5, (2005).

[3] H. Wioland, F. G. Woodhouse, J. Dunkel, J. O. Kessler, and R.E. Goldstein, Confinement stabilizes a bacterial suspension into a spiral vortex, Phys. Rev. Lett., 110, 268102, (2013).

[4] D. Saintillan, and M. J. Shelley, Instabilities, pattern formation, and mixing in active suspensions, Phys. Fluids 20, 123304, (2008).

[5] Y. Hatwalne, S. Ramaswamy, M. Rao, S. Madan and A. Simha, Rheology of active-particle suspensions, Phys. Rev. Lett. 92, 118101 (2004).

[6] S. Saha, R. Golestanian, and S. Ramaswamy, Clusters, asters, and collective oscillations in chemotactic colloids, Phys. Rev. E, 89, 062316, (2014).
[7] B. Liebchen, D. Marenduzzo, I. Pagonabarraga, and M.E. Cates, Clustering and Pattern Formation in Chemorepulsive Active Colloids, Phys. Rev. Lett. 115, 258301(2015).

[8] V. Narayan, S. Ramaswamy, and N. Menon, Long-Lived Giant Number Fluctuations in a Swarming Granular Nematic, Science 317, 105, (2007).

[9] L.J. Daniels, Y. Park, T. C. Lubensky, and D. J. Durian, Dynamics of gas-fluidized granular rods, Phys. Rev. E79, 041301(2009).

[10] A. Baskaran, and M.C. Marchetti, Hydrodynamics of self-propelled hard rods, Phys. Rev. E 77, 011920 (2008).

[11] P.G. de Gennes and J. Prost, The Physics of Liquid Crystals (Oxford University Press, Oxford, 1995)

[12] T. Vicsek, A. Czirók, E. Ben-Jacob, I. Cohen, and O. Shochet, Novel type of phase transition in a system of self-driven particles, Phys. Rev. Lett. 75, 1226, (1995).

[13] J. Toner and Y. Tu, Long-Range Order in a TwoDimensional Dynamical XY Model: How Birds Fly To- 
gether, Phys. Rev. Lett. 75, 4326 (1995).

[14] J. Toner and Y. Tu, Flocks, herds, and schools: A quantitative theory of flocking, Phys. Rev. E 58, (1998).

[15] J. Toner, Y. Tu, and S. Ramaswamy, Hydrodynamics and phases of flocks, Annals of Physics 318, 170 (2005).

[16] R.A. Simha and S. Ramaswamy, Hydrodynamic fluctuations and instabilities in ordered suspensions of selfpropelled particles, Phys. Rev. Lett. 89, 058101(2002).

[17] H. Chaté, F. Ginelli, G. Gregoire and F. Raynaud, Collective motion of self-propelled particles interacting without cohesion, Phys Rev E 77, 046113 (2008); F. Ginelli, The Physics of the Vicsek model, Eur. Phys. J. Special Topics 225, 2099 (2016).

[18] J. Toner, Giant number fluctuations in dry active polar fluids: A shocking analogy with lightning rods, J. Chem. Phys. 150, 154120 (2019).

[19] S. Ramaswamy, R.A. Simha, and J. Toner, Active nematics on a substrate: Giant number fluctuations and long-time tails, Europhys. Lett. 62, 196 (2003).

[20] C. Wolgemuth, E. Hoiczyk, D. Kaiser, and G. Oster, How myxobacteria glide, Current Biology, 12, 369 (2002).

[21] E. Lushi, and H. Wioland, and R. Goldstein, Fluid flows created by swimming bacteria drive self-organization in confined suspensions, Proceedings of the National Academy of Sciences 111, 9733 (2014).

[22] V. Schaller, and A.R. Bausch, Topological defects and density fluctuations in collectively moving systems, Proceedings of the National Academy of Sciences 110, 4488 (2013).

[23] A. Bricard,J.-B. Caussin, N. Desreumaux, O. Dauchot, and D. Bartolo, Emergence of macroscopic directed motion in populations of motile colloids, Nature 503, 95 (2013).

[24] D. Geyer, A. Morin and D. Bartolo, Sounds and hydrodynamics of polar active fluids, Nature Materials 17, 789 (2018).

[25] N. D. Mermin and H. Wagner, Absence of Ferro- magnetism or Antiferromagnetism in One- or TwoDimensional Isotropic Heisenberg Models, Phys. Rev. Lett. 17, 1133 (1966); P. C. Hohenberg, Existence of Long-Range Order in One and Two Dimensions, Phys. Rev. 158, 383 (1967); N. D. Mermin, Absence of Ordering in Certain Classical Systems, J. Math. Phys. 8, 1061 (1967).

[26] Exceptions to this result are two dimensional crystals (see, e.g., 27]) and fluctuating tethered membranes (see, e.g., [28]).

[27] B. I. Halperin and D. R. Nelson, Theory of TwoDimensional Melting, Phys. Rev. Lett. 41, 121 (1978).

[28] Y. Kantor, M. Kardar, and D. R. Nelson, Statistical Mechanics of Tethered Surfaces, Phys. Rev. Lett. 57, 791 (1986).

[29] S. Shankar, S. Ramaswamy, and M. C. Marchetti, Lownoise phase of a two-dimensional active nematic system, Phys. Rev. E 97, 012707 (2018); S. Mishra, A. Baskaran, and M. C. Marchetti, Fluctuations and pattern formation in self-propelled particles, Phys. Rev. E 81, 061916 (2010).

[30] S. Ramaswamy, R. A. Simha, and J. Toner, Active nematics on a substrate: Giant number fluctuations and long-time tails, Europhys. Lett. 62, 196 (2003).

[31] N. Sarkar, A. Basu and J. Toner, Associated long paper.

[32] These are not actually the sound speeds one would obtain from the real part of the eigenfrequencies $\omega$.

[33] Y. Zhu and S. Granick, No-Slip Boundary Condition Switches to Partial Slip When Fluid Contains Surfactant, Langmuir 18, 10058 (2002).

[34] P.C. Martin, O. Parodi, and P.S. Pershan, Unified hydrodynamic theory for crystals, liquid crystals, and normal fluids, Phys. Rev. A 6, 2401 (1972).

[35] A. Maitra, P. Srivastava, M.C. Marchetti, J.S. Lintuvuori, S. Ramaswamy, and M. Lenz, A nonequilibrium force can stabilize 2D active nematics, Proceedings of the National Academy of Sciences 115, 6934 (2018). 